\title{
Caractérisation des huiles de sept variétés d'olivier tunisiennes
}

\section{Characterization of the oils from seven Tunisian olive tree varieties}

Oléagineux, Corps Gras, Lipides. Volume 9, Numéro 2, 174-9, Mars - Juin 2002, Fondamental

Auteur(s) : Leïla ABAZA, Mongi MSALLEM, Douja DAOUD, Moktar ZARROUK, Laboratoire d'Adaptation et d'Amélioration des Plantes, INRST, B.P. 95, 2050 Hammam-Lif, Tunisie.

Author(s) : Leïla ABAZA, Mongi MSALLEM, Douja DAOUD, Moktar ZARROUK

Résumé : L'oliveraie tunisienne est riche en variétés, mais en dépit de cette richesse, aucune évaluation biochimique des huiles issues de ces variétés n'a été entreprise jusqu'à l'heure actuelle. C'est pourquoi nous procédons dans le présent travail à l'étude de la composition chimique des huiles de sept variétés à huiles cultivées en Tunisie. Outre la caractérisation variétale, cette étude a pour objectif l'amélioration de la qualité de nos huiles par le choix de variétés performantes du point de vue de la composition chimique de l'huile, pour répondre aux exigences du marché international relatives à la norme commerciale. Dans ce premier travail, nous nous intéressons à la composition de I'huile en acides gras et espèces moléculaires de triacylglycérols. Notre étude confirme qu'aussi bien la teneur en huile des olives que la composition en acides gras et triacylglycérols des huiles sont étroitement liées à la nature du cultivar. Parmi les variétés étudiées, la Sayali et la Chemchali se distinguent par un rendement élevé en huile, supérieur à $50 \%$. Ces deux variétés possèdent les taux les plus élevés en acide oléique ( 82 et $71 \%$ ) et les taux les plus bas en acide linoléique (respectivement 4 et $10 \%$ ). Elles possèdent aussi les taux les plus faibles de trilinoléine, respectivement 0,05 et 0,19\%. Par conséquent, les huiles de ces deux variétés sont à recommander pour des coupages permettant de corriger la composition chimique des huiles des autres variétés.

Summary : There are a lot of varieties in Tunisian olive plantations. However, no studies on the biochemical valuation of such varieties have been carried out until now. So, the aim of the present work is to proceed to the varietal characterization of oils from seven Tunisian varieties. To assess the chemical composition of these oils, we have considered the following in this first study: fatty acid composition and triacylglycerol molecular species. Such varietal characterization may contribute to improve the quality of our olive oils by identifying and choosing those varieties that yield oils whose chemical composition meets the requirements of the international market in terms of commercial standards and norms. Results confirm that the oil content of olives as well as the fatty acid composition or triacylglycerols molecular species are tightly linked to the type of cultivar. Among the varieties studied, Sayali and Chemchali cultivars are characterized by a high oil content (more than $50 \%$ with regards to the dry weight). Moreover, these two varieties contain the highest levels of oleic acid, 82 and $71 \%$ respectively, and the lowest levels of linoleic acid, 4 and $10 \%$ respectively. They also contain, as a consequence, the lowest levels of trilinolein (LLL), i.e. 0.05 and $0.19 \%$ respectively. So, oils from these two cultivars may be used in mixing operations to improve the chemical composition of oils from other varieties. 
Mots-clés : variétés, olivier, acides gras, triacylglycérols, caractérisation des huiles.

Keywords : olive, varieties, fatty acids, triacylglycerols, oil characterization.

\section{ARTICLE}

Bien que le patrimoine oléicole soit riche en variétés, notre oléiculture traditionnelle a été axée sur deux variétés principales : la Chétoui cultivée dans le Nord, et la Chemlali cultivée dans le Centre et le Sud du pays. Les autres variétés, dites secondaires, ont une localisation plus restreinte ou sont parfois même représentées par des pieds disséminés dans les oliveraies. En dépit de cette richesse, si des études pomologiques ont été entreprises pour la caractérisation de ces variétés [1], aucune caractérisation biochimique des huiles n'a été réalisée, surtout pour les variétés dites secondaires ou diffuses qui peuvent être potentiellement intéressantes. C'est pourquoi nous procédons, dans ce travail, à une étude de la composition chimique des huiles des sept principales variétés à huile cultivées en Tunisie.

Cette évaluation vise deux objectifs : d'une part, la caractérisation biochimique de ces variétés et, d'autre part, l'amélioration de la qualité de nos huiles pour répondre aux exigences du marché international relatives à la norme de commercialisation [2]. En effet, les huiles de la variété Chemlali de Sfax peuvent présenter des taux élevés de certains composants (acides linoléique et palmitique, trilinoléine) ou des taux bas d'acide oléique dus aux variétés actuellement utilisées. L'évaluation biochimique des huiles permettra de trouver des variétés tunisiennes qui répondent mieux aux critères exigés par la norme commerciale.

Nous nous proposons dans le présent travail de déterminer la composition acidique, la structure triglycéridique ainsi que la teneur en huile des huiles des sept variétés d'olivier étudiées.

\section{Matériel et méthodes}

\section{Matériel végétal}

Notre étude a porté sur les sept principales variétés d'olivier à huile cultivées en Tunisie : Chemchali, Chemlali, Chétoui, Gerboui, Oueslati, Sayali et Zalmati. Des arbres adultes représentant les variétés considérées sont installés dans la collection de l'Office national de l'huile $(\mathrm{ONH})$ à Béjaoua (région de Tunis), dans les mêmes conditions pédoclimatiques.

La distribution géographique des principales variétés d'olivier à huile est représentée sur la carte de la Tunisie à la figure 1. Selon cette répartition, on remarque que la variété Chemlali est la plus importante en Tunisie. Elle occupe plus des $2 / 3$ de la superficie réservée à l'oléiculture. Comme nous l'avons déjà signalé, sa culture s'étend du centre au sud du pays. La Chétoui est la deuxième principale variété à huile cultivée en Tunisie. Elle est répandue dans tout le nord du pays où elle est mélangée à d'autres variétés aussi bien à huile que de table.

À côté de ces deux principales variétés qui forment l'essentiel de l'oliveraie à huile tunisienne, nous trouvons des variétés dites secondaires cantonnées à des zones plus limitées. Ainsi, la Zalmati, variété voisine de la Chemlali, dont elle est d'ailleurs difficile à distinguer, est surtout cultivée dans 
l'extrême Sud-Est, notamment dans les régions de Zarzis, Jerba et Ben Guerdane (gouvernorat de Médnine et Tataouine). La Zalmati compte actuellement plus de 2 millions de pieds.

La Oueslati est une variété originaire des hauts plateaux. On la rencontre dans la partie septentrionale du centre de la Tunisie et en particulier à Oueslatia dans le gouvernorat de Kairouan. Quant à la Chemchali, c'est la principale variété à huile de l'oasis de Gafsa, elle se rencontre aussi au Jérid, à Tataouine et Jerba. La Gerboui est une variété anciennement cultivée dans les régions du Nord où elle côtoie le plus souvent la variété Chétoui. Actuellement, on la rencontre essentiellement dans la région du Kef.

Enfin, la Sayali est une variété présente en nombre très réduit dans les olivettes du Nord. On la trouve surtout dans les régions de Tunis et de Siliana.

\section{Méthodes d'analyse}

Deux méthodes ont été adoptées pour l'extraction des lipides totaux à partir des olives.

* La première méthode emploie un appareil Soxhlet. L'huile est extraite à l'aide de l'hexane ou de l'éther de pétrole porté à une température voisine de celle de l'ébullition du solvant, soit $60^{\circ} \mathrm{C}$ environ, et la masse d'huile est déterminée par double pesée. Cette technique est utilisée pour donner une estimation exacte de la teneur en huile des olives.

* La deuxième méthode utilise un oléodoseur et simule les conditions d'extraction dans l'industrie. L'échantillon d'olives (1,5 à $2 \mathrm{~kg}$ ) est trituré dans un broyeur à marteaux. La pâte obtenue est malaxée pendant 30 minutes puis centrifugée en couche mince pour l'extraction de l'huile. Les analyses effectuées consistent en une détermination de la composition en acides gras (AG) et en espèces moléculaires de triacylglycérols (TAG).

Les acides gras, sous forme de leurs esters méthyliques, sont analysés par chromatographie en phase gazeuse (CPG) à l'aide d'un chromatographe HP série 4890 D équipé d'une colonne capillaire Suppelcowax ${ }^{\mathrm{TM}-10} 30 \mathrm{~m} \times$ 0,53 mm de diamètre interne dont l'épaisseur du film est de 0,25 mum. La colonne est maintenue à une température isotherme de $210^{\circ} \mathrm{C}$ durant toute la durée des analyses. Les esters méthyliques sont injectés dans le chloroforme dans un injecteur de type split-splitless (division $1 / 10$ ) porté à une température de $230^{\circ} \mathrm{C}$. Ils sont détectés par un détecteur à ionisation de flamme (FID) porté à une température de $250^{\circ} \mathrm{C}$. Le débit du gaz vecteur (azote) dans la colonne est de $13 \mathrm{ml} / \mathrm{mn}$.

Les triacylglycérols purifiés par chromatographie sur couche mince de gel de silice sont fractionnés par un chromatographe HPLC de marque Waters (Milford, MA, USA) à l'aide d'une colonne C18 en phase reverse, Lichrosper $100 \mathrm{RP}-18$ dont la phase stationnaire est apolaire $(250 \times 4 \mathrm{~mm}$ de diamètre intérieur, diamètre des particules 5 mum). La colonne est placée dans une cuve où circule une eau dont la température est maintenue constante à $15^{\circ} \mathrm{C}$ grâce à un cryostat (LKB, Suisse). La phase éluante est un mélange polaire de deux solvants acétone/acétonitrile $(70 / 30, \mathrm{v} / \mathrm{v})$ à un débit de 1,4 $\mathrm{ml} / \mathrm{mn}$, en mode isocratique.

L'utilisation combinée d'une température basse de $15{ }^{\circ} \mathrm{C}$ et d'une phase éluante de polarité importante permet une meilleure résolution des espèces moléculaires de TAG. 


\section{Résultats et discussions}

Étude de quelques caractéristiques pomologiques des fruits des variétés étudiées

Bien que la caractérisation pomologique des sept variétés d'olivier qui nous intéressent dans la présente étude ait été abordée dans un travail précédent [1], nous avons jugé utile de reprendre l'analyse de deux critères se rapportant au fruit : le poids moyen de l'olive et le rapport pulpe/noyau, étant donné leur importance agronomique.

En effet, la grosseur du fruit et la proportion de la pulpe sont deux caractéristiques recherchées aussi bien pour les variétés d'olive de table que pour les variétés à huile.

La figure 2 montre les caractéristiques morphologiques des olives issues des sept variétés étudiées.

Poids moyen et pourcentage de pulpe du fruit

L'olive de la variété Sayali présente le poids frais moyen le plus élevé $(2,7 \mathrm{~g})$ suivie de près par l'olive Chétoui ( $2,47 \mathrm{~g}$ ), Chemchali ( $2,40 \mathrm{~g}$ ) et Gerboui ( $2,03 \mathrm{~g}$ ). Toutefois, les olives Zalmati, Chemlali et Oueslati sont plus petites et présentent un poids moyen qui ne dépasse pas $1 \mathrm{~g}$ (figure 3 ).

Le fruit Chétoui semble être le plus riche en pulpe, présentant un pourcentage de pulpe de 90,3\%, alors que le fruit Chemlali est le moins charnu, avec un pourcentage de pulpe de $81,7 \%$. Les fruits des autres variétés présentent un pourcentage de pulpe intermédiaire compris entre 83,3 et $88,2 \%$ (figure 4).

Considérant le poids moyen des olives, deux groupes de variétés se distinguent. Le premier est représenté par les cultivars ayant des fruits de grosseur moyenne (poids moyen supérieur ou égal à 2 g) qui sont la Sayali, la Chétoui, la Chemchali et la Gerboui. Le second groupe est représenté par les variétés à petits fruits (poids moyen inférieur ou égal à $1 \mathrm{~g}$ ); il s'agit des variétés Zalmati, Chemlali et Oueslati.

Comparées à la Meski - une variété de table de grande réputation dont le poids moyen du fruit est de $6,5 \mathrm{~g}$ - les sept variétés étudiées ont des fruits relativement petits à moyens, d'où leur classification comme variétés à huile, bien que les variétés Chemchali et Sayali soient souvent considérées comme des variétés à double aptitude.

La description pomologique des fruits montre déjà des différences entre les variétés étudiées, ne serait-ce que de point de vue pondéral. En est-il de même de l'analyse biochimique des variétés ?

Évaluation des huiles des variétés étudiées

\section{* Teneur en huile}

La teneur en huile des olives ne constitue pas un critère de détermination de la qualité de l'huile mais c'est surtout un critère à envisager lors d'une sélection variétale.

La teneur en huile exprimée en pourcentage de matière fraîche oscille entre 16,8 et 24,4 \% ; exprimée en pourcentage de matière sèche, elle varie entre 40,6 et $55,1 \%$ selon les variétés (figure $5)$. 
La variété Sayali présente le rendement en huile le plus élevé par rapport à la matière fraîche $(24,4$ \%) suivie des variétés Gerboui et Chétoui. Les teneurs les plus élevées par rapport à la matière sèche sont observées pour les variétés Chétoui, Chemchali et Sayali (autour de $51 \%$ ). Les variétés Chemlali, Zalmati et Oueslati présentent des teneurs en huile plus faibles, voisines de $40 \%$. La variété Gerboui se situe dans une position intermédiaire entre les deux groupes de variétés.

Tous et Romero, cités par Sànchez et al. [3], ont classé les variétés selon leur rendement en huile par rapport à la matière sèche comme suit :

- variétés présentant un rendement élevé (> $46 \%$ ) ;

- variétés présentant un rendement moyen (de 38 à $46 \%$ ) ;

- variétés présentant un rendement faible (<38\%).

D'après cette classification, on peut dire que les variétés Chétoui, Chemchali, Sayali et Gerboui sont à teneur élevée en huile, tandis que les autres variétés Chemlali, Zalmati et Oueslati sont à teneur moyenne en huile dans les conditions de culture de la station de l'Office national de l'huile à Béjaoua.

\section{* Composition en acides gras}

L'étude de la composition acidique des huiles des diverses variétés permet de distinguer les acides gras suivants (tableau 1) :

- des acides gras saturés : acide palmitique (C16:0), acide stéarique (C18:0) et acide arachidique (C20:0) ;

- des acides gras monoinsaturés : acide palmitoléique (C16:1) et acide oléique (C18:1) ;

- un acide gras diinsaturé : I'acide linoléique (C18:2) ;

- un acide gras triinsaturé : I'acide linolénique (C18:3). Cette composition en acides gras de I'huile d'olive est analogue à celle observée par d'autres auteurs [4-6].

L'examen du tableau 1 montre également que la composition en acides gras des huiles d'olive testées est variable et nettement influencée par le facteur variétal.

On remarque principalement que le taux de l'acide oléique, acide gras majeur de l'huile d'olive, est le plus faible pour la Gerboui $(58,8 \%)$ alors qu'il est le plus élevé pour la Sayali $(81,7 \%)$; il a une valeur intermédiaire de $71,1 \%$ pour la variété Chemchali alors que, pour les Chemlali, Chétoui, Oueslati et Zalmati, ce taux oscille entre 60 et $68 \%$.

Le pourcentage le plus élevé de l'acide linoléique est observé chez la Gerboui $(22,5 \%)$ et le plus bas chez la Sayali (3,7\%). Les autres variétés présentent des taux compris entre 15 et $19 \%$, sauf pour la variété Chemchali dont le taux est de $10,3 \%$.

Les huiles de la Zalmati et de la Chemlali sont les plus riches en acide palmitique, dont les taux respectifs sont de 18,4 et 17,9\%, ce qui rend ces huiles figeables à température relativement basse. 
Le pourcentage de ce même acide gras n'est que de $11 \%$ dans le cas de la Sayali et de la Oueslati, alors qu'il varie de 12,4 à $14 \%$ pour les autres variétés (tableau 1 ).

Les autres acides gras ( $C 16: 1, C 18: 0, C 18: 3$ et $C 20: 0)$ sont faiblement représentés, bien que leurs taux changent en fonction du facteur variétal.

La proportion des acides gras saturés (AGS) est variable ; elle oscille entre 13,4 (Sayali) et 21,1 \% (Zalmati). De la même manière, le pourcentage des acides gras insaturés (AGI) varie légèrement en fonction des variétés. Il est de l'ordre de $80 \%$ pour toutes les variétés, mais le taux le plus important est enregistré chez la Sayali (86,6\%).

II est à remarquer que, mis à part la variété Gerboui qui présente un taux en acide linoléique (C18:2) de l'ordre de 22,5\% (supérieur à la valeur limite de la norme), toutes les huiles des variétés d'olivier considérées ont des compositions en acides gras conformes à la norme de commercialisation.

La détermination de la composition en acides gras des huiles étudiées révèle la performance de la variété Sayali qui se distingue des autres par un taux d'acide oléique élevé $(81,7 \%)$, des pourcentages en acides linoléique et palmitique bas (respectivement de 3,7 et $11 \%$ ) et une prédominance nette d'acides gras insaturés $(86,6 \%)$ par rapport aux acides gras saturés qui ne représentent que $13,4 \%$ des acides gras totaux (AGT).

\section{* Composition en espèces moléculaires de triacylglycérols (TAG)}

L'analyse des espèces moléculaires de triacylglycérols, composants majeurs de l'huile d'olive et principaux lipides de réserve, montre une grande variabilité de cette fraction en fonction des variétés.

L'examen du tableau 2 permet de distinguer essentiellement treize espèces moléculaires de TAG dans l'huile d'olive. Les plus importantes sont la trioléine (OOO), la dioléopalmitine (POO), la dioléolinoléine (OOL) et la palmitooléolinoléine (POL). Ces résultats sont en accord avec ceux d'autres auteurs [6-8].

Pour toutes les variétés d'olivier étudiées, les huiles sont caractérisées par l'abondance de la trioléine (OOO) dont le taux est très élevé dans le cas de la Sayali (51,73 \%) et faible chez la Zalmati, la Gerboui et la Chemlali, ne dépassant pas $25 \%$. Pour les trois autres variétés - Chemchali, Chétoui et Oueslati ce triglycéride varie entre 31,91 et $36,41 \%$.

La structure triglycéridique des huiles des différentes variétés montre, en plus, la présence de grandes quantités de POO (17,10 à $24,13 \%)$ et $\mathrm{OOL}(8,08$ à $21,82 \%)$. Les huiles d'olive tunisiennes apparaissent alors très riches en acide oléique et pauvres en acides linoléique, linolénique, stéarique et palmitique.

La présence dans une huile d'olive de taux élevés de triglycérides comportant l'acide oléique et inversement de taux faibles de triglycérides avec de l'acide linoléique constitue un indice favorable de l'authenticité. Mais c'est plutôt la détermination du taux de la trilinoléine (LLL) qui constitue un 
paramètre pouvant être utilisé pour identifier l'ajout de faibles quantités d'huiles de graines à l'huile d'olive $[7,9,10]$.

Dans le cas de nos huiles, la teneur en LLL change suivant les variétés : elle est supérieure à 0,5\% (limite de LLL fixée par la norme) pour les variétés Oueslati, Gerboui et Chétoui, alors qu'elle est inférieure à cette valeur pour les quatre autres. De plus, il est à remarquer que, pour la Sayali, ce triglycéride est extrêmement faible, il ne réalise que $0,05 \%$, soit le $1 / 10^{\mathrm{e}}$ de celui de la variété Chétoui (tableau 2).

\section{Discussion}

Nos résultats permettent de noter que la teneur en huile des olives est dépendante de la variété. Des études récentes ont montré que le contenu en huile du fruit est très influencé par l'humidité de la pulpe au moment de la récolte du fruit [3]. II est alors plus juste d'examiner le rendement en huile par rapport à la matière sèche que par rapport à la matière fraîche. Ainsi, et en se référant à la classification de Tous et Romero cités par Sànchez et al. [3], les variétés tunisiennes étudiées peuvent être classées en deux catégories :

- variétés à rendement élevé en huile $>46 \%$ : c'est le cas des variétés Chétoui, Sayali, Gerboui et Chemchali (figure 5);

- variétés à rendement moyen en huile compris entre 38 et $46 \%$; on peut classer dans ce groupe les variétés Oueslati, Zalmati et Chemlali (figure 5). De la même manière, la composition en acides gras des huiles d'olives analysées est largement influencée par le facteur variétal. C'est ainsi que la variété Sayali se distingue des autres variétés par le taux le plus élevé de C18:1 (81,71\%), le pourcentage le plus faible de $\mathrm{C} 18: 2$ ne dépassant même pas $4 \%$ et un taux de $\mathrm{C} 16: 0$ relativement bas (11 \%). À côté de la variété Sayali, une autre se distingue du lot : c'est la variété Chemchali qui a une composition caractérisée par un pourcentage relativement élevé en acide oléique (C18:1) représentant $71,1 \%$ des acides gras totaux et un taux relativement bas d'acide linoléique (C18:2) n'excédant pas $10 \%$. Le taux d'acide palmitique n'est pas élevé (14\%) (tableau 1).

En considérant les taux de $\mathrm{C} 18: 1$ et $\mathrm{C} 18: 2$, les huiles peuvent être groupées en huiles à teneur faible à moyenne en $\mathrm{C} 18: 1$, ce qui correspond à une teneur forte à moyenne en C18:2 - c'est le cas des huiles des variétés Chemlali, Chétoui, Gerboui, Oueslati et Zalmati -, et en huiles à teneur forte en C18:1, soit une faible teneur en C18:2 - c'est le cas des huiles des variétés Chemchali et Sayali (tableau 1).

La première catégorie des huiles définies correspond bien à celle des huiles rencontrées en Afrique du Nord, alors que la seconde ressemble à celle des huiles européennes [8].

Le changement de la composition en acides gras de l'huile d'olive en fonction du paramètre variétal a été également observé chez d'autres variétés étrangères et par d'autres auteurs $[3,7,11,12]$.

Il faut également signaler que la composition en acides gras des huiles varie en fonction de l'origine géographique et de la zone de production de l'huile (climat, latitude) bien que cela ne semble pas être toujours vérifié [13-15]. C'est pourquoi il est intéressant de comparer la composition des huiles produites dans les sites d'origine des variétés avec celle des huiles issues des oliviers de la station de Béjaoua ; ce sera l'objet d'une prochaine étude. Kachouri et al. [16] ont montré, en revanche, que 
des variétés européennes Arbéquine et Leccino installées dans la station expérimentale de Borj-El Amri (dans la région de Tunis) ont une composition en acides gras identique à celle de leur site d'origine. Dans le même ordre d'idée, des travaux récents de Deidda et al. [17] sur la qualité de I'huile d'olive en rapport avec les conditions climatiques ont montré qu'il n'existe aucune corrélation entre la température et la qualité de l'huile. L'étude de la structure triglycéridique des huiles des sept variétés étudiées a permis d'identifier treize espèces moléculaires de TAG, l'espèce majoritaire dans presque toutes les variétés est la trioléine (OOO) suivie de la dioléopalmitine (POO), la dioléolinoléine $(\mathrm{OOL})$ et la palmitooléolinoléine $(\mathrm{POL})$. Les autres espèces sont relativement faiblement représentées (tableau 2).

Les proportions des diverses fractions triglycériques sont variables selon les cultivars. Le taux de trioléine, par exemple, varie de $51,7 \%$ chez la Sayali à $21,5 \%$ chez la Zalmati. Les autres variétés ont des taux intermédiaires de trioléine (tableau 2). Ces observations sont en quelque sorte attendues puisque la composition acidique de ces mêmes huiles varie en fonction de la nature des variétés.

Toutefois, parmi les treize espèces moléculaires de triacylglycérols isolées, la trilinoléine (LLL) peut permettre de détecter le mélange de I'huile l'olive avec de faibles quantités d'huiles de graines, essentiellement des huiles linoléiques.

\section{CONCLUSION}

La norme de commercialisation a fixé la limite supérieure de trilinoléine à $0,5 \%$, ce qui permet de détecter par exemple de faibles ajouts d'huile de tournesol (jusqu'à $2 \%$ ) dans l'huile d'olive [8, 9]. La limite supérieure en LLL imposée par la norme commerciale paraît faible pour certaines huiles tunisiennes, comme c'est le cas pour les huiles des variétés Oueslati, Gerboui et Chétoui. Cependant, dans les huiles de la variété Sayali, le taux de trilinoléine n'excède pas $0,05 \%$, soit le $1 / 10^{\mathrm{e}}$ de la valeur fixée par la norme. L'huile de cette variété peut servir pour des coupages éventuels avec les huiles des variétés à teneur élevée en trilinoléine comme la Oueslati, la Gerboui, ou la Chétoui.

Par conséquent, pour améliorer la qualité de nos huiles et valoriser notre oléiculture, les deux variétés Sayali et Chemchali sont à prendre en considération pour les nouvelles plantations d'oliviers ou pour le remplacement des vieilles oliveraies. Les huiles de ces deux variétés sont aussi à recommander pour des procédés de coupage afin d'améliorer la composition des huiles des autres variétés. Toutefois, la plantation de ces deux variétés en dehors de leur zone de culture nécessite au préalable une étude de leur comportement agronomique ainsi que du rendement et de la qualité de leurs huiles.

\section{REFERENCES}

1. CONSEIL OLÉICOLE INTERNATIONAL (1998). Norme commerciale applicable à I'huile d'olive et à I'huile de grignons d'olive. COI/T.15/NC n²/Rév. 8, 25 novembre 1998.

2. MEHRI H, HELLALI R (1995). Étude pomologique des principales variétés d'olivier cultivées en Tunisie. Numéro spécial, Institut de l'Olivier, Tunisie, $45 \mathrm{p}$. 
3. SÀNCHEZ CASAS J, DE MIGUEL GORDILLO C, MARIN EXPOSITO J (1999). La qualité de l'huile d'olive provenant de variétés en Estrémadure en fonction de la composition et la maturation de l'olive.

Olivae, $79: 38-40$.

4. ZARROUK M, CHÉRIF A (1984). Action du chlorure de sodium sur la composition en acides gras de I'olive. In : BEN HAMIDA F, ed. Actes de la $4^{e}$ École Franco-africaine de biologie moléculaire. Paris : IJM/CNRS ; 537-40.

5. FEDELI E (1997). Technologie de production et de conservation de I'huile. In : Conseil Oléicole International, ed. Encyclopédie Mondiale de l'Olivier. Madrid (Espagne) : COI : 251-91.

6. MORDRET F (1999). Conférence Chevreul : Evolution des critères de qualité des huiles d'olive vierges - Perspectives. OCL, $6: 69-76$.

7. OLLIVIER D, SOUILLOL S, GUÉRERE M, PINATEL C, ARLAUD J (2000). Données récentes sur la composition en acides gras et en triglycérides d'huiles d'olive vierges françaises. Nouvel Olivier, 13 : 13-8.

8. UZZAN A (1992). L'huile d'olive. In : Manuel des Corps Gras. Paris : Lavoisier : 221-8.

9. CORTESI N (1993). La chromatographie liquide haute performance (HPLC) dans l'analyse de l'huile. Olivae, $45: 40-2$.

10. MINCIONE B, GIUFFRE AM, LEUZZI U, LOMBARDO N (1994). Ricerche sugli oli di oliva monovarietali, Nota I Caratterizzazione della produzione della Calabria. Riv Ital Sostanze Grasse, 71 : 335-8.

11. CERT A, ALBA J, CAMINO P (1999). Influence des systèmes d'extractions sur les caractéristiques et les composés mineurs de l'huile d'olive vierge extra. Olivae, 79 : 41-50.

12. TISCORINA E, FIORINO N, EVANGELISTI F (1982). Chemical composition of olive oil and variations induced by refining. Riv Ital Sostanze Grasse, 59 : 519-56.

13. PANNELLI G, SERVILI M, SELVAGGINI R, BALDIOLI M, MONTEDORO GF (1994). Effect of agronomic and seasonal factors on olive (Olea europaea L.) production and on the qualitative characteristics of the oil. Acta Hortic, 356 : 239-43.

14. APARICIO R, FERRERO L, ALONSO V (1994). Effect of climate on the chemical composition of vergin olive oil. Anal Chem Acta, 292 : 235-41.

15. MOUSSA MY, GERASOPOULOS D (1996). Effect of altitude on fruit and oil quality characteristics of « Mastoides » olives. J Sci Food Agric, $71: 345-50$.

16. KACHOURI M, M'SALLEM M, ZARROUK, M, CHÉRIF A (1995). Comparative study of oil composition in four olive varieties. In : KADER JC, MAZLIAK P, eds. Plant Lipid Metabolism. Dordrecht : Kluwer Academic Publishers : 567-9.

17. DEIDDA P, NIEDDU G, SPANO D, et al. (1994). Olive quality in relation to environmental conditions. Acta Hortic, 356 : 354-7. 


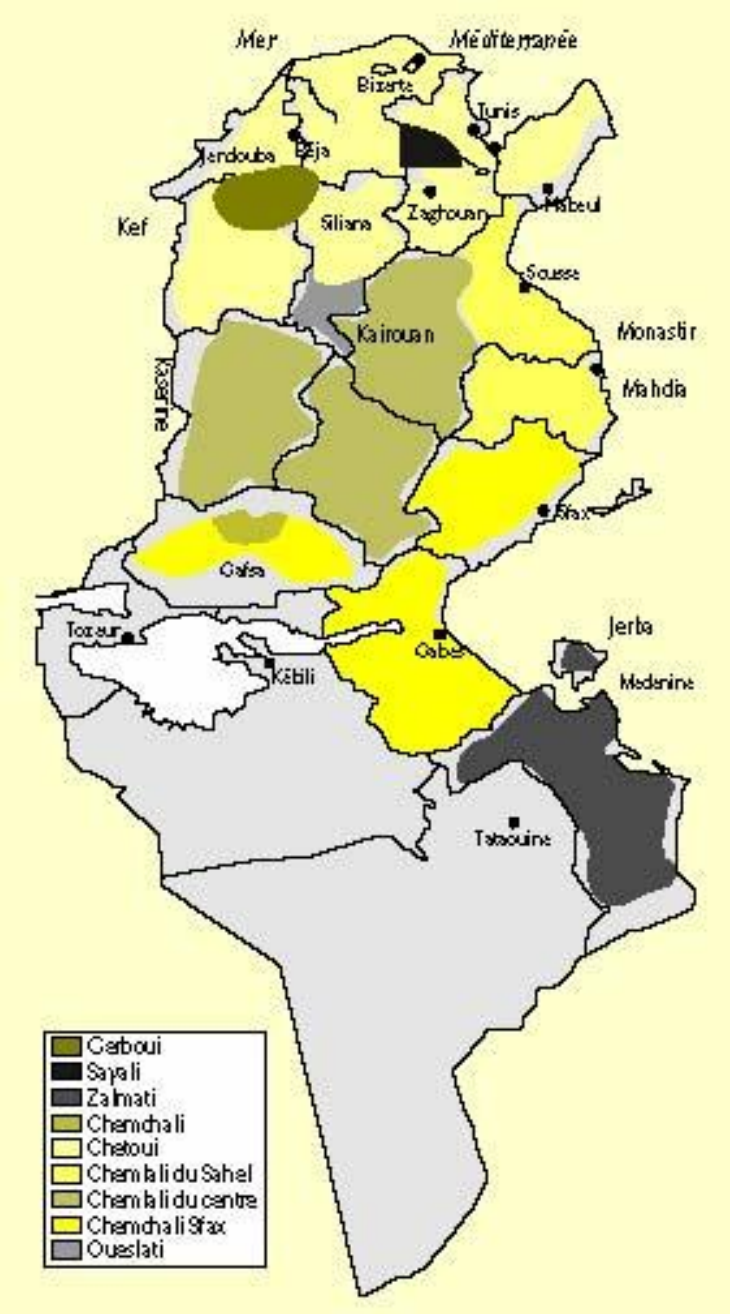

Figure 1. Répartition des principales variétés d'olivier à huile en Tunisie (Msallem 1999) (d'après I'Institut de l'olivier, 1999). 


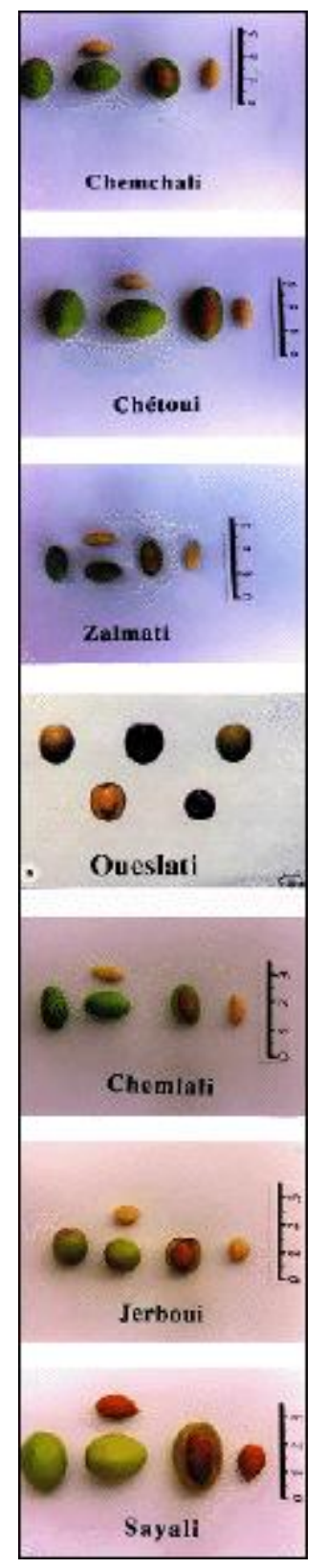

Figure 2. Morphologie des olives et des noyaux des variètés d'olivier étudiées. 


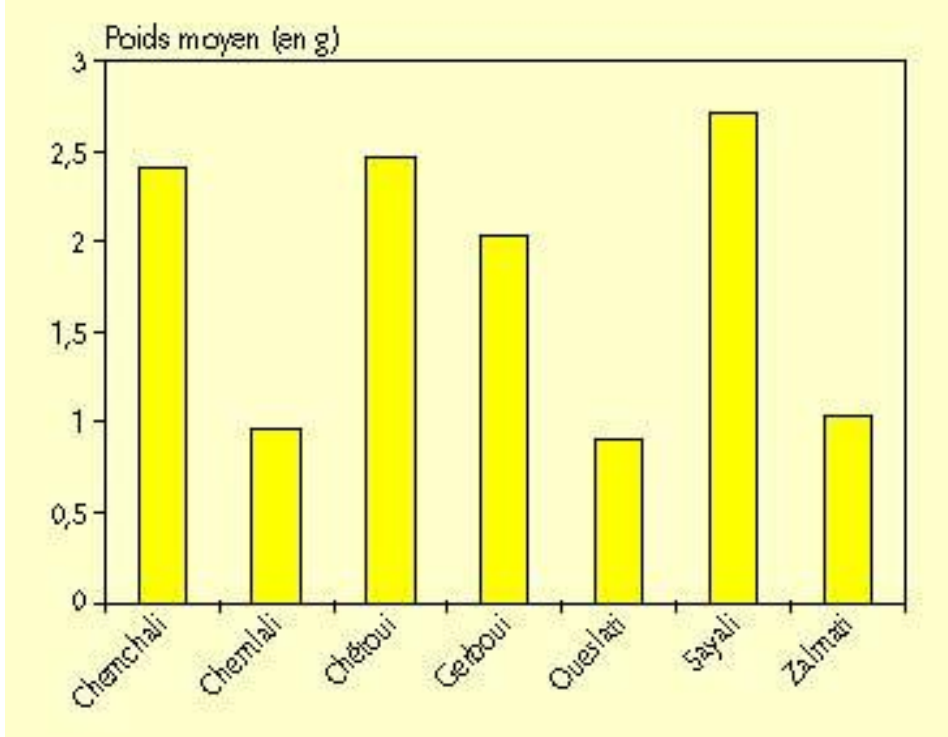

Figure 3. Poids moyen des olives des sept variétés d'olivier étudiées.

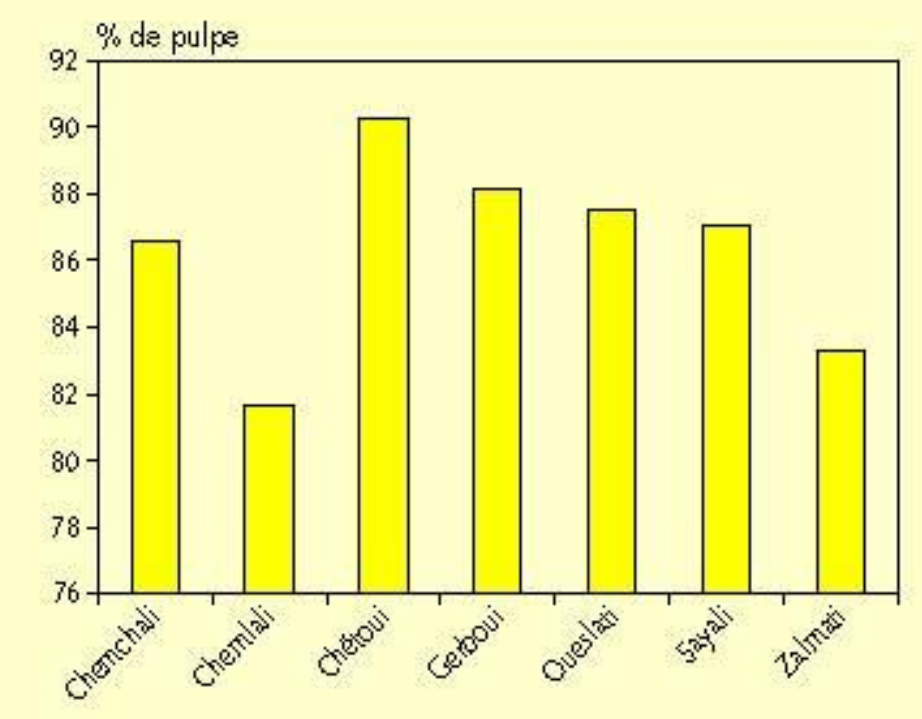

Figure 4. Pourcentage de pulpe des olives des sept variétés d'olivier considérées. 


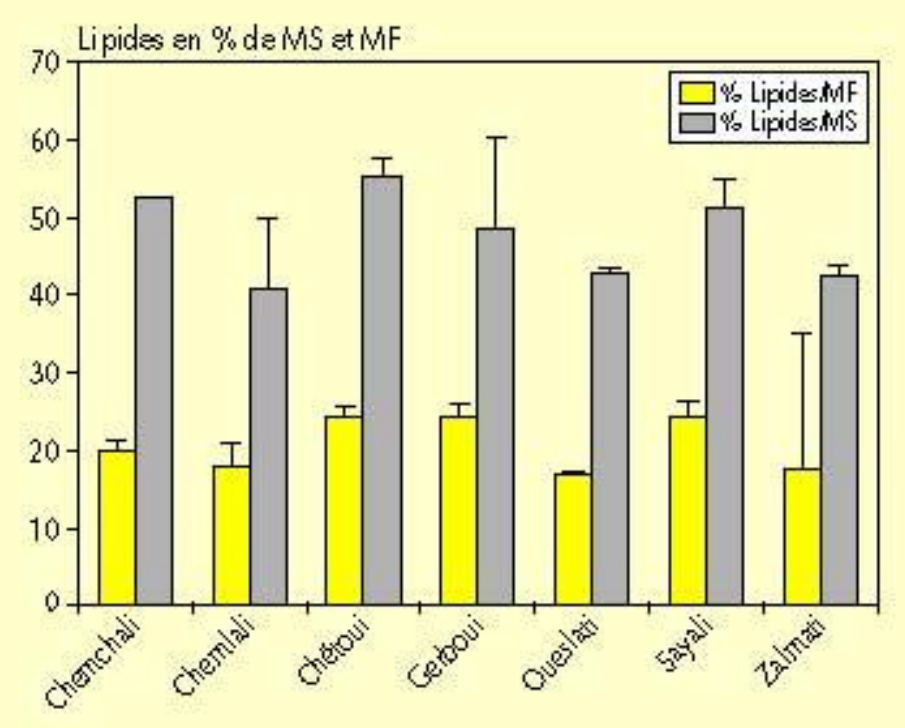

Figure 5. Teneur en huile des olives des sept variétés d'olivier étudiées.

Tableau 1. Composition en acides gras des huiles des sept va iétés d'o livier étudiée (en \% des acides gas totaux, AGT) (Norme : norme du Conseil oléicole inter natio nal (CO, 1998)).

\begin{tabular}{|l|cccccccc}
\hline & \multicolumn{7}{|c}{ Variétés } \\
\hline $\begin{array}{l}\text { \% en } \\
\text { AGT }\end{array}$ & Norme & Chemchali & Chemlali & Chétoui & Gerboui & Oueslati & Sayali & Zalm ati \\
\hline $16: 0$ & $7,5-20$ & 14 & 17,9 & 12,4 & 13,9 & 11,2 & 11 & 18,4 \\
$16: 1$ & $0,3-3,5$ & 0,9 & 2,3 & 0,7 & 0,7 & 0,6 & 0,6 & 1,9 \\
$18: 0$ & $0,5-5$ & 2,7 & 2,2 & 3 & 2,9 & 2,7 & 2 & 2,2 \\
$18: 1$ & $55-83$ & 71,1 & 61,3 & 63,1 & 58,8 & 67,9 & 81,7 & 60,5 \\
$18: 2$ & $3,5-21$ & 10,3 & 15,1 & 19 & 22,5 & 16,6 & 3,7 & 15,6 \\
$18: 3$ & $<0,9$ & 0,5 & 0,7 & 0,6 & 0,8 & 0,6 & 0,6 & 0,8 \\
$20: 0$ & $\leq 0,6$ & 0,5 & 0,4 & 0,4 & 0,4 & 0,4 & 0,4 & 0,5 \\
AGI & - & 82,8 & 79,5 & 83,5 & 82,8 & 85,4 & 86,6 & 78,9 \\
ACS & - & 17,2 & 20,5 & 15,9 & 17,1 & 14,3 & 13,4 & 21,1 \\
\hline
\end{tabular}


Tableau 2. Composition en espèces moléculaires de TAG des huiles des variêtés d'olivier étudiées (en \% des TAG totaux). LLL : 0,5 \% selon la norme (Uzzan, 1992).

\begin{tabular}{|l|ccccccc|}
\hline & \multicolumn{7}{|c}{ Variétés } \\
\hline $\begin{array}{l}\text { Esp. Molé } \\
\text { TAC }\end{array}$ & Chemchali & Chemlali & Chétoui & Gerboui & Oueslati & Sayali & Zalm ati \\
\hline LLL & 0,2 & 0,3 & 0,5 & 0,5 & 0,7 & 0,05 & 0,4 \\
OLL $_{n}$ & 0,2 & 0,5 & 0,2 & 0,3 & 0,4 & 0,2 & 0,5 \\
LLO & 2,6 & 5,0 & 6,4 & 9,9 & 6,4 & 0,04 & 6,7 \\
OLL $_{n}$ & 0,9 & 1,6 & 0,80 & 1 & 1,10 & 0,6 & - \\
PLO & 0,8 & 2,2 & 1,10 & 3,1 & 1,5 & 1,2 & 2,3 \\
PLL & 0,5 & 1,1 & 0,2 & 0,8 & 0,5 & 0,1 & 1,1 \\
OOL & 15,4 & 15,9 & 21,8 & 20,10 & 21,5 & 8,1 & 18,2 \\
POL & 7,8 & 12,9 & 7,8 & 12,7 & 9,3 & 2,5 & 13,20 \\
OOO & 36,4 & 24,6 & 34,4 & 22,2 & 31,9 & 51,7 & 21,5 \\
POO & 22,9 & 22,6 & 17,10 & 17,5 & 18 & 24,1 & 22,6 \\
SOO & 4,8 & 5,8 & 2,3 & 4,80 & 2,9 & 4 & 5,8 \\
SPO & 4,6 & 3 & 5,2 & 3,7 & 4 & 4,5 & 3,01 \\
AOO & 1,3 & 1,2 & 1,0 & 1,2 & 0,9 & 0,9 & 1,1 \\
\hline
\end{tabular}

